The AstrophySICAL JoURNAL, 399:L55-L58, 1992 November 1

(C) 1992. The American Astronomical Society. All rights reserved. Printed in U.S.A.

\title{
HIGH SPATIAL RESOLUTION CONTINUUM AND H $\alpha$ IMAGING OF THE HIGH-REDSHIFT IRAS SOURCE FSC $10214+4724$
}

\author{
B. T. Solfer, ${ }^{1}$ G. Neugebauer, ${ }^{1}$ K. Matthews, ${ }^{1}$ ANd C. Lawrence ${ }^{2}$ \\ Palomar Observatory, California Institute of Technology, Pasadena, CA 91125 \\ AND \\ J. Mazzarella ${ }^{3}$ \\ Infrared Processing and Analysis Center, California Institute of Technology, Pasadena, CA 91125 \\ Received 1992 June 25; accepted 1992 August 12
}

\begin{abstract}
High spatial resolution images have been obtained of the high-redshift $(z=2.286)$ IRAS source FSC $10214+4724$ at wavelengths corresponding to the optical continuum and $\mathrm{H} \alpha$ in the object's rest frame. The continuum shows two clumps separated by $\sim 1^{\prime \prime}(\sim 10 \mathrm{kpc})$ and aligned approximately north-south. The structure appears similar at rest wavelengths of $0.38,0.64$, and $0.67 \mu \mathrm{m}$. The continuum morphology suggests two separate concentrations of luminosity, likely the nuclei of interacting galaxies, although a large galaxy bifurcated by an immense dust lane cannot be ruled out. The southern component is predominantly extended in the east-west direction on a scale of $\sim 0.5(5 \mathrm{kpc})$, consistent with the position and elongation of the radio source reported in 1992 by Lawrence et al.

The $\mathrm{H} \alpha$ emission is centered on the southern component of the galaxy and is unresolved with a diameter smaller than $0.4(<3.5 \mathrm{kpc})$. A simple explanation for the current observations is an interacting system comprised of two or more highly luminous $\left(\sim 10^{12} L_{\odot}\right)$ galaxies, one of which contains a dust enshrouded quasar that generates the bulk of the bolometric luminosity $\left(\sim 5 \times 10^{14} L_{\odot}\right)$ of the system.
\end{abstract}

Subject headings: galaxies: formation - galaxies: interactions

\section{INTRODUCTION}

The IRAS source FSC $10214+4724$ was identified with a faint optical galaxy at a redshift of $z=2.286$ (Rowan-Robinson et al. 1991). FSC $10214+4724$ is the most luminous object known in the universe $\left(L \sim 5 \times 10^{14} L_{\odot}\right)$, about a factor of 2 more luminous than the most luminous known quasar. The identification of the IRAS source was confirmed by the detection of the $J=3-2$ line of CO at the optical redshift (Brown \& Vandenbout 1991) and by the detection of the continuum at 450 and $850 \mu \mathrm{m}$ (Clements et al. 1992). The molecular gas content $\left(\sim 6 \times 10^{11} M_{\odot}\right.$ for $\left.H_{0}=75 \mathrm{~km} \mathrm{~s}^{-1} \mathrm{Mpc}^{-1}\right)$ exceeds by an order of magnitude that of the next most massive known molecular gas-rich galaxy (Solomon, Radford, \& Downes 1992). The infrared luminosity to gas mass ratio is several hundred in solar units (Solomon et al.), an order of magnitude larger than that found in active star forming galaxies in the local universe (Sanders, Scoville, \& Soifer 1991). The large infrared luminosity, if produced via thermal emission from dust, requires $\gtrsim 10^{8.5} M_{\odot}$ of dust.

In this Letter, we present observations that show that the underlying structure of the galaxy associated with FSC $10214+4724$ is like that of interacting galaxies or high-redshift radio galaxies, while the dominant ionizing and dust heating source is likely to be associated with a dust enshrouded quasar. At the redshift of FSC 10214+4724, 1" corresponds to $\sim 10$ $\mathrm{kpc}$ for either $H_{0}=75 \mathrm{~km} \mathrm{~s}^{-1} \mathrm{Mpc}^{-1}$ and $q_{0}=0$ or $H_{0}=50$ $\mathrm{km} \mathrm{s}^{-1} \mathrm{Mpc}^{-1}$ and $q_{0}=0.5$.

\footnotetext{
${ }^{1}$ California Institute of Technology, 320-47, Pasadena, CA 91125

${ }^{2}$ California Institute of Technology, 105-24, Pasadena, CA 91125.

3 California Institute of Technology, 100-22, Pasadena, CA 91125.
}

\section{OBSERVATIONS AND DATA REDUCTION}

Near-infrared images of FSC $10214+4724$ were obtained using the Cassegrain camera on the 200 inch Hale telescope of the Palomar Observatory. Broad-band observations were obtained at $J(1.27 \mu \mathrm{m}$ [rest wavelength $0.386 \mu \mathrm{m}])$ and $K(2.2$ $\mu \mathrm{m}$ [rest wavelength $0.670 \mu \mathrm{m}$ ]) with spectral resolution of $\Delta \lambda / \lambda \sim 0.2$. Narrow-band images with $\Delta \lambda / \lambda=0.013$ were also obtained at wavelengths of $2.124,2.156$, and $2.190 \mu \mathrm{m}$, corresponding to rest wavelengths of $0.646,0.656$, and $0.666 \mu \mathrm{m}$, respectively. The camera uses a $58 \times 62 \mathrm{InSb}$ array and has a plate scale of 0.313 pixel $^{-1}$ corresponding to a field of view of $18^{\prime \prime} \times 19^{\prime \prime}$. The 1.27 and $2.2 \mu \mathrm{m}$ images used standard $J$ and $K$ filters, while the narrow-band images were obtained through a circular variable filter.

Approximately 20 minutes of exposures were obtained with each of the broad-band filters and in excess of $8 \mathrm{hr}$ were obtained with the narrow-band filters including and bracketing the $\mathrm{H} \alpha$ line. On all nights the seeing at $2 \mu \mathrm{m}$ was $\sim 0 " 7-1{ }^{\prime \prime} 0$ full width at half-maximum. Between frames the telescope was moved in the north-south direction to avoid bad pixels and so that successive frames could be used for sky subtraction. Accurate tracking of the telescope was maintained through the use of an offset autoguider.

In order to determine the point spread function and the location of the sources, all the images contained a nearby field star (star A in the notation of Rowan-Robinson et al. 1991). About half of the images were taken with the camera rotated $180^{\circ}$ so that any irregularities in the camera and array were shared equally by images of the star and galaxy. Shifting the different frames for proper co-addition was accomplished using the known offsets to the offset guide star. Each pixel was subdivided into 100 pixels, and the pixel values were bilinearly interpolated between the centers of the original pixels. 


\section{RESULTS}

The galaxy and star A are displayed in the contour plots of Figure 1, showing the galaxy in broad-band light at $0.38 \mu \mathrm{m}$ I II (hereafter we refer to the various observations by the rest wavelength) as well as in the narrow-band filters at $0.656 \mu \mathrm{m}$, i.e., the $\mathrm{H} \alpha$ line, and in the combined adjacent narrow-band continua $(0.646 \mu \mathrm{m}+0.666 \mu \mathrm{m})$. The continuum emission (Fig. $1 b$ ) is seen to be broken into at least two distinct blobs. At 0.67 $\mu \mathrm{m}$ broad-band (not shown) the southern source is dominated by $\mathrm{H} \alpha$ emission but the demarcation between the northern and southern components is also evident.

Based on the spectrum presented by Soifer et al. (1991), there are no significant contributions from emission lines in the continuum image at $0.646 \mu \mathrm{m}$, while the [S II] lines at $0.672 \mu \mathrm{m}$ may contribute to the image at $0.666 \mu \mathrm{m}$. Within the signal-tonoise ratio of the images there is no discernable difference between the two narrow-band continuum images. The spectrum of Soifer et al. showed a rest frame equivalent width of $\mathrm{H} \alpha+[\mathrm{N} \mathrm{II}]$ of $0.07 \pm 0.02 \mu \mathrm{m}$. This implies that the line contributes $\sim 90 \%$ of the flux in the $0.656 \mu \mathrm{m}$ narrow-band image. Based on case B recombination theory (see Osterbrock 1989) the contribution of the high-level Balmer lines in the broadband $J$ image should be small; however, the bandpass of this filter includes an unknown contribution from the [O II] 0.3727 $\mu \mathrm{m}$ line.
Figure 2 shows cuts through the sources in the 0.646 $\mu \mathrm{m}+0.666 \mu \mathrm{m}$ continuum and cuts through a model consisting of two images of star A superposed to match the spacing and relative brightnesses of the north-south components of the galaxy. It is seen that the northern component of the galaxy is probably unresolved, while the southern component appears to be resolved, having an extent of $\sim 0.5(5 \mathrm{kpc})$ mostly in the east-west direction. There is a suggestion that the southern source can be represented by two point sources spaced $\sim 0$ ". 5 apart, but it is beyond the resolution of the current images to distinguish between two point sources and an extended source.

The location of the peak of the southern source in the 0.646 $\mu \mathrm{m}+0.666 \mu \mathrm{m}$ continuum is $1.28 \pm 0.01$ west and $0.2 \pm 0.1$ south of star A, leading to a position of R.A. $=10^{\mathrm{h}} 21^{\mathrm{m}} 31^{\mathrm{s}} .10$ \pm 0.03 , decl. $=+47^{\circ} 24^{\prime} 23^{\prime \prime} .4 \pm 0^{\prime \prime} .3$ (1950) for the southern peak of the galaxy based on the position of star A (C. J. Lonsdale, private communication). This is within 0.1 of the radio position determined by Lawrence et al. (1992). The uncertainties are primarily in the position of star A. This implies a direct association of the radio source with the southern component of the infrared continuum source, an association which is strengthened by the apparent elongation of the southern continuum source primarily in the east-west direction, consistent with the size and orientation of the radio continuum source reported by Lawrence et al.

The image of pure $\mathrm{H} \alpha$, derived by subtracting the sum of the
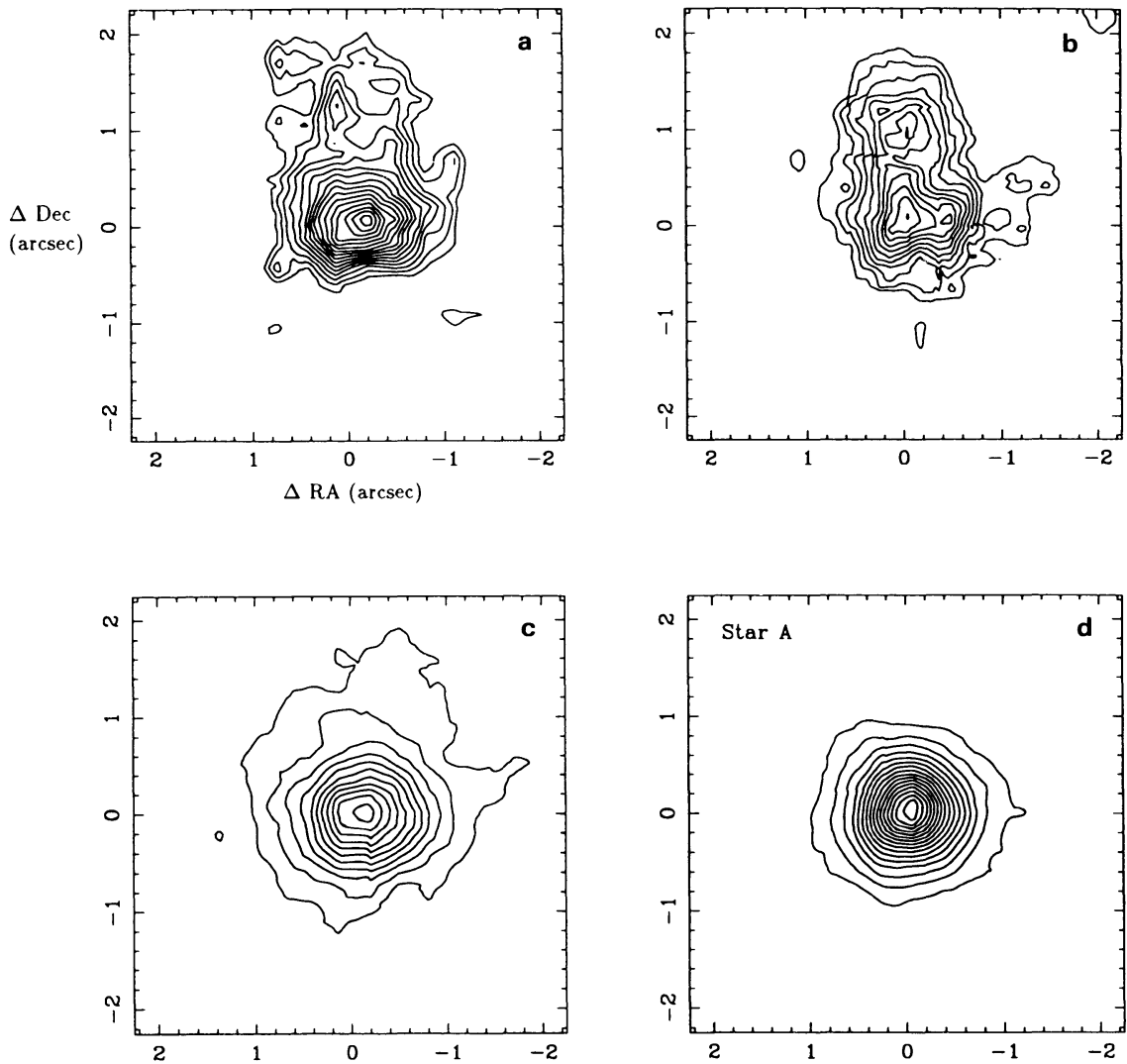

Fig. 1.-Contour plots of images in (a) broad-band $J$ continuum (rest wavelength $0.386 \mu \mathrm{m}$ ), (b) narrow-band continuum at 2.124 and $2.190 \mu \mathrm{m}$ (rest wavelengths $0.646 \mu \mathrm{m}+0.666 \mu \mathrm{m}$ ) bracketing $\mathrm{H} \alpha$ in the rest frame, and (c) narrow-band at $2.156 \mu \mathrm{m}$ (H $\alpha$ in the rest frame) of FSC 10214 +4724 . (d) Narrow-band continuum image for reference star A (in the same frame as the image of $(b)$. North is up and east to the left, with axes labeled in arcseconds offset from the peak of the southern narrow-band $(0.646 \mu \mathrm{m}+0.666 \mu \mathrm{m})$ continuum component. 

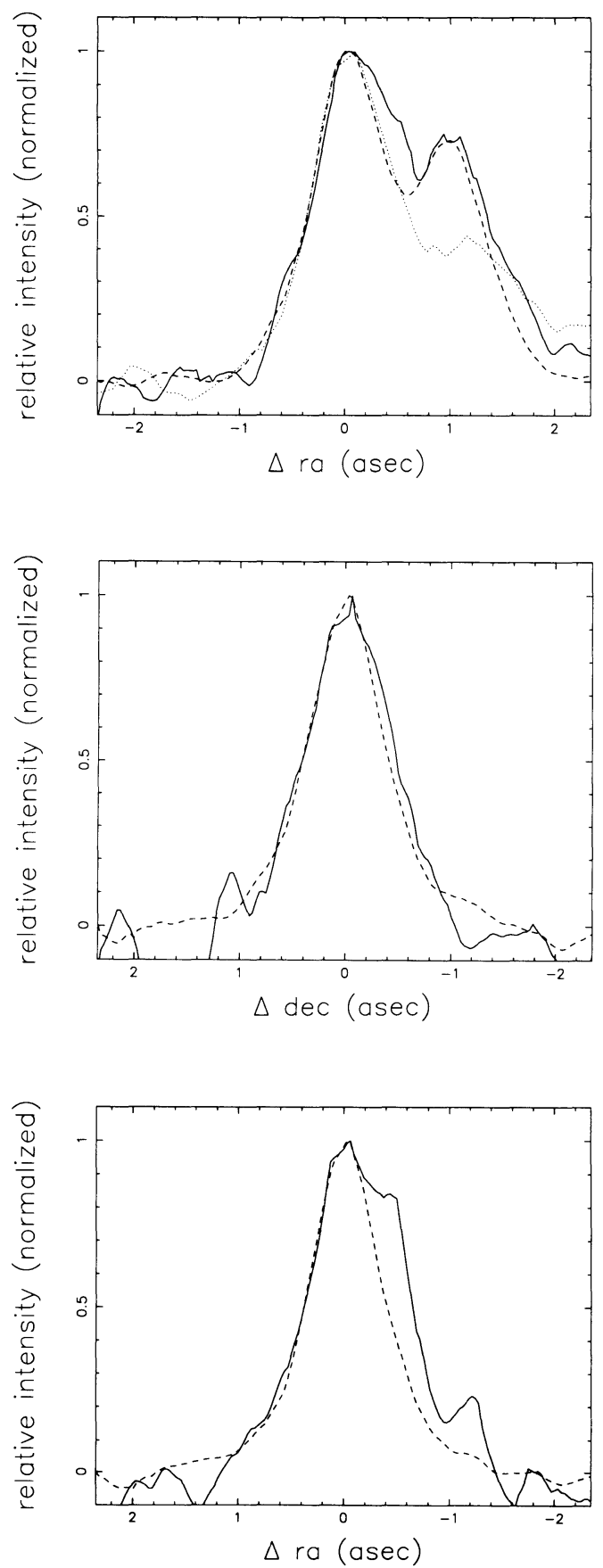

FIG. 2.-Plots of the intensity in a 0 " 34 wide declination strip through the main peak of the galaxy (top) and a right ascension strip through the southern peak (bottom) and through the northern peak (middle) of the $0.646 \mu \mathrm{m}+0.666$ $\mu \mathrm{m}$ continuum image are shown as solid lines. The corresponding strips through a model consisting of two superposed images of star A (taken from the same frames as the galaxy) separated by $1^{\prime \prime}$ are indicated by the dashed lines. The model position was adjusted to get the best appearing fit to the data. A declination scan through the observed peaks at $0.386 \mu \mathrm{m}$ is given by the dotted line in the top panel. Each scan has been arbitrarily normalized to the peak intensity.

adjacent narrow-band continuum images from the narrowband image at the wavelength of $\mathrm{H} \alpha$, is pointlike and centered within 0.1 of the eastern component of the southern source seen in Figure 1. The pointlike nature is illustrated in Figure 3, which shows cuts through the $\mathrm{H} \alpha$ image of the galaxy and the image of star A seen at $0.65 \mu \mathrm{m}$ and the adjacent continuum. Models convolving the star A image with Gaussian profiles set an upper limit of 0 ". 4 for the intrinsic width of any $\mathrm{H} \alpha$ source.

The total extent of the galaxy is not well determined by the observations reported here. Photometry in the broad-band images at $0.67 \mu \mathrm{m}$ shows flux still increasing to an aperture diameter of 4". This is consistent with the optical and $2.2 \mu \mathrm{m}$ imaging of Rowan-Robinson et al. (1991) and Lawrence et al. (1992). Thus the galaxy halo appears to be of substantial size.

\section{DISCUSSION AND SPECULATION}

The continuum image at $0.646 \mu \mathrm{m}+0.666 \mu \mathrm{m}$ (Fig. $1 b$ ) shows a double structure with a north-south separation. The image at $0.38 \mu \mathrm{m}$ (Fig. 1a) also shows extended structure in the north-south direction. Since the wavelengths of the images are significantly different, this suggests that either the structure is due to distinct condensations or nuclei within the galaxy, or the galaxy is cut by an immense, highly opaque dust lane, at least 0.3 or $3 \mathrm{kpc}$ wide, giving the system the appearance of having distinct components. Such an appearance is given by optical imagery of Arp 220 (e.g., Sanders et al. 1988). Figure 2 also contains a cut in declination through the $0.38 \mu \mathrm{m}$ image. The fact that the contrast of the double structure at $0.38 \mu \mathrm{m}$ is less than at $0.646 \mu \mathrm{m}+0.666 \mu \mathrm{m}$ favors the explanation that
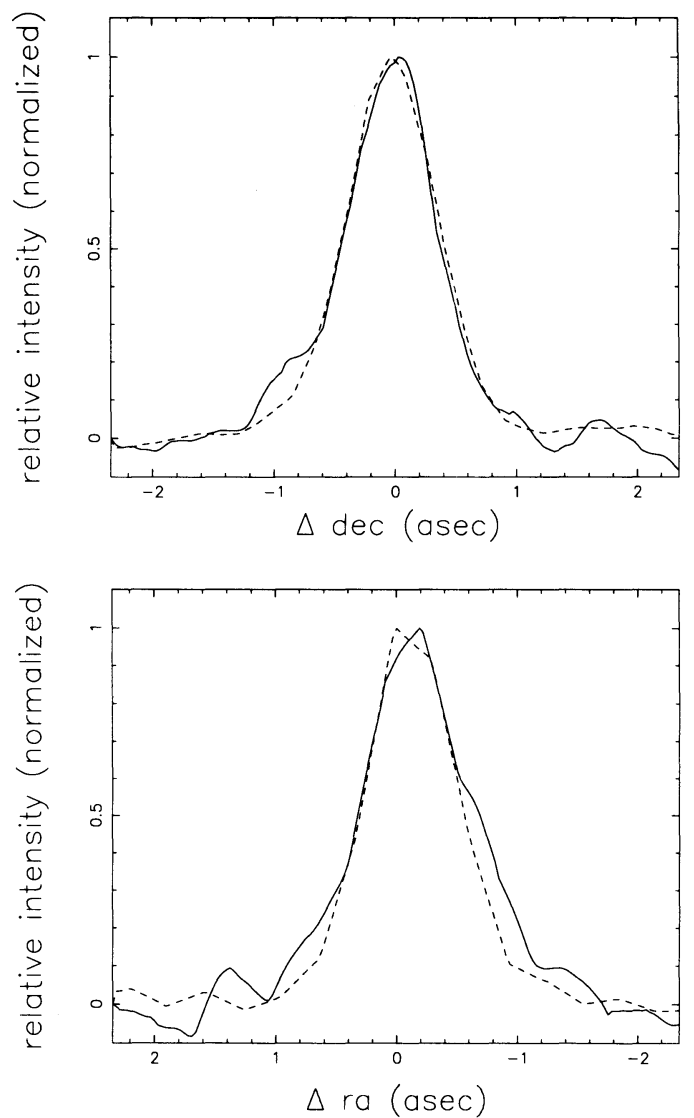

Fig. 3.-Plots of the intensity in a 0"34 wide declination strip (top) and a 0 " 34 wide right ascension strip (bottom) through the $\mathrm{H} \alpha$ image are given by the solid lines. The $\mathrm{H} \alpha$ image was produced by subtracting the $0.646 \mu \mathrm{m}+0.666$ $\mu \mathrm{m}$ continuum narrow-band image from the $0.656 \mu \mathrm{m}$ image. The dashed curves show the corresponding scans over the star image in the same frames obtained by adding the $0.646 \mu \mathrm{m}$ and $0.666 \mu \mathrm{m}$ continuum and $0.656 \mu \mathrm{m}$ narrow-band images. 
these are distinct nuclei, since an absorption band should be more pronounced at the shorter wavelength.

The best-studied galaxies at high redshifts are radio galaxies. These systems often show highly peculiar morphologies (e.g., McCarthy, Persson, \& West 1992c; Eisenhardt \& Chokshi 1990). The data presented in Figure 1, particularly the continuum image at $0.646 \mu \mathrm{m}+0.666 \mu \mathrm{m}$, show a complex structure for FSC $10214+4724$ that is similar to that seen in many of these high-redshift galaxies, with the substantial difference being the high (rest frame) optical luminosity of FSC $10214+4724$ compared to radio galaxies. The observed 0.67 $\mu \mathrm{m}$ continuum luminosity, uncorrected for extinction, is $\sim 2 \times 10^{12} L_{\odot}$. As pointed out originally by Rowan-Robinson et al. (1991), and emphasized by Soifer et al. (1991) and Lawrence et al. (1992), FSC $10214+4724$ is more than 2 mag brighter than predicted based on the $K$-band Hubble diagram for radio galaxies (Lilly 1989). With extinction, the intrinsic luminosity could be as much as an order of magnitude greater than this. The similar brightnesses of the two components imply that each is a highly luminous galactic nucleus. The possibility that the northern source is simply a projection effect seems highly unlikely since the individual sources have $K$ magnitudes of $\sim 17$.

The peculiar structure, coupled with the high luminosities of the individual clumps suggest that the system is actually an interacting system comprised of two or more extremely luminous galaxies. The high luminosities argue that each galaxy is in an early stage of evolution. Although the colors of FSC $10214+4724$ are quite typical of those seen in radio galaxies at similar redshifts (McCarthy et al. 1992c; Eisenhardt \& Chokshi 1990), the uncertainty in the reddening allows a purely hot star continuum if the extinction is $A_{v} \sim 3$ mag.

The coincidence of the southern continuum component source with the $H \alpha$ source and the radio source suggests that the bulk of the bolometric luminosity of this system is generated in the southern component. The compact nature of the $\mathrm{H} \alpha$ source, with a surface brightness at least four orders of magnitude greater than seen in the extended Ly $\alpha$ nebulosity of most high-redshift radio galaxies (McCarthy, Elston \& Eisenhardt 1990a; McCarthy et al. 1992b) suggests the picture in which the $\mathrm{H} \alpha$ emission originates in the narrow-line region of a central quasar (the observations of Eisenhardt, McCarthy, \& Elston [1992] show that the $\mathrm{H} \alpha$ line is unresolved with a line width of $\lesssim 1000 \mathrm{~km} \mathrm{~s}^{-1}$ ). If the models of Phinney (1989) are scaled to the luminosity of FSC $10214+4724$, such emission would emerge from a region of size $\sim 300 \mathrm{pc}$ and would thereby remain spatially unresolved in the current observations. The central source that ionizes such a region would also be easily obscured by an extended dust cloud. In such an environment, dust would have to be at a distance of $2-4 \mathrm{kpc}$ to reach the observed temperature of $\sim 150 \mathrm{~K}$ (Rowan-Robinson et al. 1991). A large mass of dust and gas of such a size could cause the apparent structure seen in the southern source.

The alternative picture of a starburst origin for the $\mathrm{H} \alpha$ nebulosity poses several obvious problems. First, if the starburst is confined to the size of the $\mathrm{H} \alpha$ source, i.e., less than 0.4 or 3.5 $\mathrm{kpc}$, the luminosity density must be $\gtrsim 10^{3}-10^{4} L_{\odot} \mathrm{pc}^{-3}$ over this entire volume. Indeed, it is surprising that a starburst of such a magnitude would be confined to such a small size, considering the extent of the radio source and the size of the entire galaxy. Additionally, the continuum associated with this ionizing radiation would have to be obscured so as to not dominate the observed $0.65 \mu \mathrm{m}$ continuum or be totally comprised of hot stars to generate the bolometric luminosity observed. The latter possibility is testable with a high signal-tonoise-ratio (rest frame) UV spectrum.

The total gas mass associated with FSC $10214+4724$ cannot be confined to the compact $\mathrm{H} \alpha$ structure observed here. The observed linewidth of $300 \mathrm{~km} \mathrm{~s}^{-1}$ (Brown \& Vanden Bout 1991) implies that the vast majority of the $\sim 10^{12} M_{\odot}$ (Solomon, Radford, \& Downes 1992) of gas must be at a distance of less than $20 \mathrm{kpc}$. The dust required to radiate the observed far infrared luminosity, i.e., $\sim 4 \times 10^{8} M_{\odot}$ of dust or $\sim 4 \times 10^{10} M_{\odot}$ of gas, could be at a distance of $2-4 \mathrm{kpc}$ from the heating source, and thus associated with the compact $\mathrm{H} \alpha$ source observed here.

The quasar must be heavily obscured to preclude its being viewed directly. This picture also suggests that the strong observed polarization (Lawrence et al. 1992) is light scattered from the dust enshrouded quasar. The extensive gas cloud at a distance of $\sim 10-30 \mathrm{kpc}$ could provide substantial extinction of the entire galaxy system, and lead to a substantial fraction of the galaxy's luminosity being radiated in the far infrared, and submillimeter (Lawrence et al. 1992).

In summary, these new observations support the view that FSC $10214+4724$ is a rare, extreme example of the class of ultraluminous infrared galaxies observed in the local universe (e.g., Sanders et al. 1988). The presence of two continuum components and a compact $(D \lesssim 3.5 \mathrm{kpc}) \mathrm{H} \alpha$ source suggests that a merger between two or more gas-rich systems has triggered the production of a powerful dust-enshrouded quasar which is responsible for the bulk of the luminosity.

We thank Juan Carasco and Lee Armus for assistance with the observations and E. S. Phinney and M. Voit for helpful discussions. This research was supported in part by grants from the NSF and NASA. IPAC is supported by the Jet Propulsion Laboratory, California Institute of Technology, under contract with NASA.

\section{REFERENCES}

Brown, R. L., \& Vanden Bout, P. A. 1991, AJ, 102, 1956

Clements, D. L., Rowan-Robinson, M., Lawrence, A., Broadhurst, T., \& McMahon, R. 1992, MNRAS, 256, 35

Eisenhardt, P., \& Chokshi, A. 1990, ApJ, 351, L9

Eisenhardt, P., McCarthy, P., \& Elstor, R. 1992, in preparation

Lawrence, A., et al. 1992, MNRAS, in press

Lilly, S. 1989, ApJ, 340, 77

Mathis, J. S. 1990, ARA\&A, 28, 37

McCarthy, P. J., Elston, R., \& Eisenhardt, P. 1992a, ApJ, 387, L29

McCarthy, P. J., Kapahi, V. K., Van Breugel, W., \& Subrahmanya, C. R. 1990b, AJ, 100, 1014

McCarthy, P. J., Persson, S. E., \& West, S. C. 1992c, ApJ, 386, 52
McCarthy, P. J., Spinrad, H., Van Breugel, W., Liebert, J., Dickinson, M., Djorgovski, S., \& Eisenhardt, P. 1990b, ApJ, 365, 487

Osterbrock, D. E. 1989, Astrophysics of Gaseous Nebulae and Active Galactic Nuclei (Mill Valley, CA: University Science Books)

Phinney, S. 1989, in Theory of Accretion Disks, ed. P. Meyer, W. Duschl, J. Frank, \& E. Meyer-Hofmeister (Dordrecht: Kluwer), 451 Rowan-Robinson, M., et al. 1991, Nature, 351, 719

Sanders, D. B., Soifer, B. T., Elias, J. H., Madore, B. F., Matthews, K., Neugebauer, G., \& Scoville, N. Z. 1988, ApJ, 325, 74

Sanders, D. B., Scoville, N. Z., \& Soifer, B. T. 1991, ApJ, 370, 158

Soifer, B. T., et al. 1991, ApJ, 381, L55

Solomon, P. M., Radford, S. J. E., \& Downes, D. 1992, Nature, 356, 318 